\title{
La modélisation par Reverse Monte Carlo (RMC)
}

\author{
R.L. McGreevy
}

ISIS Facility, Rutherford Appleton Laboratory, Chilton, Didcot, Oxon OX11 OQX, U.K.

\begin{abstract}
Résumé : La technique de modélisation par Reverse Monte Carlo (RMC) est une méthode générale de modélisation structurale à partir d'un ensemble de données expérimentales. Cette méthode étant très souple, elle peut s'appliquer à de nombreux types de données. Jusqu'à présent ces applications comprennent : la diffraction des neutrons (y compris la substitution isotopique), la diffraction des rayons $\mathrm{X}$ ( $\mathrm{y}$ compris la diffusion anomale), la diffraction des électrons, la RMN (les techniques d'angle magique et de 2ème moment) et l'EXAFS. Les systèmes étudiés sont également d'une grande variété : liquides, verres, polymères, cristaux et matériaux magnétiques, par exemple. Ce cours présente les bases de la méthode RMC en signalant certaines des idées fausses répandues. L'accent sera mis sur le fait que les modèles structuraux obtenus par RMC ne sont ni 'uniques' ni 'exacts' ; cependant ils sont souvent utiles à la compréhension soit de la structure du système, soit des relations entre structure et autres propriétés physiques.
\end{abstract}

\section{INTRODUCTION}

Les propriétés physiques des matériaux étant déterminées par leur structure atomique, l'obtention de données expérimentales concernant la structure puis l'élaboration de modèles structuraux basés sur ces données sont fondamentales pour l'étude de la matière condensée. Pendant une grande partie du 20 ème siècle l'accent a été mis sur les matériaux cristallins et de nombreuses méthodes ont été développées afin d'élaborer ou d'affiner des modèles de la structure moyenne (dans le temps) des cristaux à partir des données expérimentales. En revanche, la compréhension de la structure des matériaux non-cristallins (e.g. les liquides, verres, polymères), dont l'importance technologique égale sinon dépasse celle des matériaux cristallins, est encore relativement primitive et peu de méthodes de modélisation structurale ont été développées.

La modélisation par Reverse Monte Carlo (RMC) est une méthode générale de modélisation structurale basée sur des données expérimentales [1]. Le RMC a été initialement développé afin de modéliser la structure des liquides et des verres, mais actuellement il est également appliqué aux structures cristallines et magnétiques [2-5]. Beaucoup de types de données peuvent être utilisés et de nombreux systèmes peuvent être modélisés par RMC [6-13]. Bien qu'il y ait actuellement plus de 400 articles publiés utilisant les méthodes $\mathrm{RMC}$, on entend encore des commentaires comme 'Le RMC ne marche pas' ou peut-être 'Je n'y crois pas'. De telles réactions sont les conséquences de certaines idées fausses encore répandues. Le RMC est une méthode de modélisation structurale. Son but est d'élaborer un modèle, ou bien un ensemble de modèles, qui soit cohérent avec les données disponibles et avec d'autres informations fournies sous forme de contraintes. Il n'est pas judicieux de poser la question 'Est-ce le bon modèle?' puisque la réponse ne pourra jamais être connue. On devrait par contre se demander 'Est-ce un modèle valable?'. Est-ce que ce modèle 
peut nous aider à mieux comprendre les relations entre la structure et une propriété physique particulière, voire même nous aider à proposer de nouvelles expériences?

Ce cours décrit la méthode RMC classique ainsi que quelques-unes de ses variations. L'algorithme RMC ayant déjà été décrit en détail $[3,5,6,9-13]$, ne seront exposées ici que les grandes lignes de la méthode mais de façon suffisamment approfondie pour permettre au lecteur non-initié de comprendre l'intégralité de ce cours. Certaines des idées reçues et critiques du RMC seront discutées plus en détail, bien qu'elles soient souvent plutôt philosophiques que scientifiques. Le lecteur est également invité à lire un récent article de revue sur plusieurs applications de la modélisation RMC [14].

Même si la méthode RMC est critiquée, son plus grand succès est sans aucun doute d'avoir démontré que les modèles structuraux tri-dimensionnels des matériaux désordonnés pouvaient être élaborés à partir des données expérimentales. Ce succès a stimulé le développement d'autres méthodes voisines ou dérivées du RMC, elles seront également discutées dans ce cours. Il est désormais admis que le RMC ne concurrence pas les méthodes de simulation numérique telles que le Monte Carlo ou la Dynamique Moléculaire, mais qu'il est plutôt complémentaire de cellesci. Le RMC insiste sur l'importance des modèles structuraux tri-dimensionnels, mais aussi sur l'importance des détails des données expérimentales dans la compréhension des matériaux réels (à opposer aux matériaux 'modèles').

\section{LA METHODE RMC}

\subsection{L'essentiel de l'algorithme RMC}

Le RMC est lui-même une variante de la méthode standard de Metropolis Monte Carlo (MMC) [15]. Le but est d'élaborer un modèle structural (i.e. un ensemble d'atomes, habituellement appelé une configuration) qui soit cohérent avec une ou plusieurs séries de données expérimentales (aux incertitudes de mesure près) et soumis à un ensemble de contraintes. Les erreurs de mesures sont supposées aléatoires avec une distribution statistique normale (cela sera discuté ultérieurement plus en détail). La méthode est brièvement exposée ici dans le cas de données de diffraction de neutrons par un liquide ou un verre. L'extension de la méthode à d'autres types de données a déjà été décrite ailleurs [e.g. 5]. Le logiciel le plus répandu exploitant l'algorithme décrit ci-dessous est appelé RMCA [9].

1. Placer $N$ atomes dans une boîte avec des conditions aux limites périodiques - c'est à dire, la bô̂te est entourée par des images d'elle-même. Normalement une boîte de forme cubique est choisie, bien que d'autres géométries soient possible (par exemple, une super-boîte comprenant plusieurs mailles d'un cristal). Il vaut mieux que les 3 dimensions de la boîte soient aussi égales que possible. La densité d'atomes devrait avoir si possible la même valeur que celle expérimentale. Les positions initiales des atomes sont arbitraires: soit aléatoires, soit provenant d'une structure cristalline ou des résultats d'un autre modèle ou simulation. L'ensemble des coordonnées des atomes et des dimensions de la boite s'appelle une configuration.

2. Calculer la fonction de distribution de paires partielle (ici aussi appelée la fonction de distribution radiale partielle) pour cette configuration initiale :

$$
g_{\alpha \beta}^{C o}(r)=\frac{n_{\alpha \beta}^{C o}(r)}{4 \pi r^{2} d r \rho c_{\beta}}
$$


où $\rho$ est la densité d'atomes et $c_{\beta}$ la concentration d'atomes de type $\beta$. Le nombre de coordination (ou coordinence) $n_{\alpha \hat{Q}}^{C o}(r)$ est le nombre d'atomes de type $\beta$ à une distance entre $r$ et $r+d r$ d'un certain atome de type $\alpha$, puis moyenné sur tous les atomes de type $\alpha$ dans la boîte. L'exposant ' $\mathrm{C}$ ' veut dire 'calculé' et l'exposant 'o' veut dire 'old' (ancien).

3. Effectuer une transformée de Fourier des $g_{\alpha \beta}^{C o}(r)$ pour obtenir les facteurs de structure partiels,

$$
A_{\alpha \beta}^{C o}(Q)-1=\rho \int_{0}^{\infty} 4 \pi r^{2}\left[g_{\alpha \beta}^{C o}(r)-1\right] \frac{\sin (Q r)}{Q r} d r
$$

où $Q$ est le vecteur de diffusion.

4. Calculer le facteur de structure total

$$
F^{C o}(Q)=\sum_{\alpha, \beta} c_{\alpha} c_{\beta} b_{\alpha} b_{\beta}\left[A_{\alpha \beta}^{C o}(Q)-1\right]
$$

où $b_{\alpha}$ est la longueur de diffusion cohérente des neutrons pour un atome de type $\alpha$.

5. Calculer la différence entre le facteur de structure total mesuré $F^{E}(Q)$ et celui calculé à partir de la configuration $F^{C o}(Q)$ de la façon suivante :

$$
\chi_{o}^{2}=\sum_{i=1}^{m}\left[F^{C o}\left(Q_{i}\right)-F^{E}\left(Q_{i}\right)\right]^{2} / \sigma^{2}\left(Q_{i}\right)
$$

où la somme est sur les $m$ points expérimentaux et $\sigma$ représente l'incertitude expérimentale. Notons que la valeur minimale de $Q_{i}$ devrait être supérieure ou égale à $2 \pi / L$ où $L$ est la dimension minimale de la configuration.

6. Déplacer aléatoirement un seul atome (en pratique il y a une distance de déplacement minimale). Si la distance séparant 2 atomes est inférieure à une distance minimale prédéfinie (i.e. une distance de coupure ou 'cut-off'), le déplacement est rejeté et un nouvel atome est choisi pour un nouvel essai de déplacement.

7. Calculer les fonctions de distribution radiale partielles de la nouvelle configuration $g_{\alpha \beta}^{C n}(r)$, ainsi que les nouveaux facteurs de structure partiels $A_{\alpha \beta}^{C n}(Q)$ et le nouveau facteur de structure total $F^{C n}(Q)$, puis la différence :

$$
\chi_{n}^{2}=\sum_{i=1}^{m}\left[F^{C n}\left(Q_{i}\right)-F^{E}\left(Q_{i}\right)\right]^{2} / \sigma^{2}\left(Q_{i}\right)
$$

8. Si $\chi_{n}^{2}<\chi_{o}^{2}$ le déplacement est accepté, i.e. la 'nouvelle' configuration devient 'l'ancienne' configuration. Si $\chi_{n}^{2}>\chi_{o}^{2}$ le déplacement est accepté avec la probabilité $\exp \left[-\frac{1}{2}\left(\chi_{n}^{2}-\chi_{o}^{2}\right)\right]$, autrement il est rejeté.

9. Répéter la procédure à partir de l'étape 6 .

$\mathrm{Au}$ fur et à mesure que le nombre de déplacements acceptés augmente, la quantité $\chi^{2}$ diminue pour approcher une valeur d'équilibre autour de laquelle elle continuera à varier. La configuration ainsi obtenue représente donc une structure tri-dimensionnelle qui est cohérente avec les données expérimentales aux erreurs près. Ici nous avons choisi de fitter le facteur de structure $F^{E}(Q)$ mesuré, alors que la fonction de distribution de paires (déterminée par les données expérimentales) 
aurait pu être choisie. La figure 1 illustre quelques étapes dans la modélisation RMC d'un système simple.

Dans la procédure Metropolis Monte Carlo (MMC), la quantité minimisée lors de l'affinement est $\left(U_{n}-U_{o}\right) / k T$, où $U$ est l'énergie potentielle de la configuration calculée à partir d'un potentiel interatomique donné, $T$ la température et $k$ la constante de Boltzmann. Dans le RMC, c'est donc le facteur de structure qui joue le rôle de l'énergie de la configuration, i.e. il 'conduit' l'affinement, et $\sigma$ joue le rôle de la température. La définition exacte de $\chi^{2}$ dans le RMC n'est en fait pas très importante. Le but du MMC est de produire une configuration ayant une distribution Boltzmann d'énergies, tandis que le but du RMC est de produire une configuration cohérente avec les données dans la limite des erreurs expérimentales dont les erreurs systématiques (de distribution inconnue) sont généralement les plus importantes. La forme de $\chi^{2}$ est choisie pour le RMC car l'analogie avec le MMC est commode, et parce que ca marche!

Il est utile de rappeler quelques idées clefs de l'algorithme RMC qui sont à l'origine de son succès par rapport à d'autres méthodes [e.g. 16-19] :

1. Les conditions aux limites périodiques évitent les effets de bord, et en pratique la grande taille des configurations (au moins plusieurs milliers d'atomes) évite également les effets de petit nombre.

2. Puisque certains déplacements augmentant la valeur de $\chi^{2}$ sont acceptés, la configuration finale peut être (en principe) indépendante de la configuration initiale.

3. Lorsque cela est possible, il est préférable d'affiner directement les données expérimentales (e.g. le facteur de structure total), plutôt que des fonctions dérivées des données (e.g. les fonctions de distribution radiale partielles) ou bien un ensemble de paramètres dérivés des données (e.g. des paramètres d'ordre à courte distance $[18,19]$ ). Cette procédure d'affinement direct des données rend plus facile la compréhension des effets des erreurs expérimentales sur la configuration obtenue. $\mathrm{Si}$, par exemple, on dispose d'un jeux de 3 facteurs de structure totaux mesurés par diffraction de neutrons avec substitution isotopique (NDIS) et que l'un d'entre eux est faussé par des erreurs expérimentales importantes, ceci se révélera tout de suite lors de l'affinement RMC. Par contre, si l'affinement est réalisé sur les 3 fonctions de distribution radiales partielles correspondantes, toutes seront 'contaminées' par le facteur de structure total faussé, l'algorithme aura donc du mal à identifier le problème de mauvaise convergence de l'affinement et la configuration obtenue sera également faussée.

4. Le RMC n'utilise pas de potentiel interatomique dans le sens conventionnel du terme, mais seulement dans l'aspect trivial d'une distance minimale interatomique qui joue en fait le rôle d'un potentiel de sphères dures. Le RMC peut donc s'appliquer à n'importe quel système. D'autres aspects concernant une éventuelle utilisation de potentiels interatomiques dans le RMC seront discutés ultérieurement. L'absence d'un potentiel a le désavantage de priver les modèles RMC d'une cohérence thermodynamique. D'un autre coté, l'utilisation d'un potentiel pourrait donner lieu à des contradictions : si le potentiel choisi est fixé et qu'il n'est pas compatible avec les données, il est alors possible de minimiser soit $\chi^{2}$ soit l'énergie de la configuration, mais non tous les deux. Si le potentiel lui-même est affiné pour être cohérent avec les données, il faut que la forme fonctionnelle du potentiel soit appropriée. Si la mauvaise forme du potentiel est choisie (e.g. un potentiel de paires lorsque les termes de $n$ corps sont significatifs), l'affinement risque de conduire à un mauvais résultat. L'utilisation des potentiels sera davantage discutée dans la section 5.3 . 

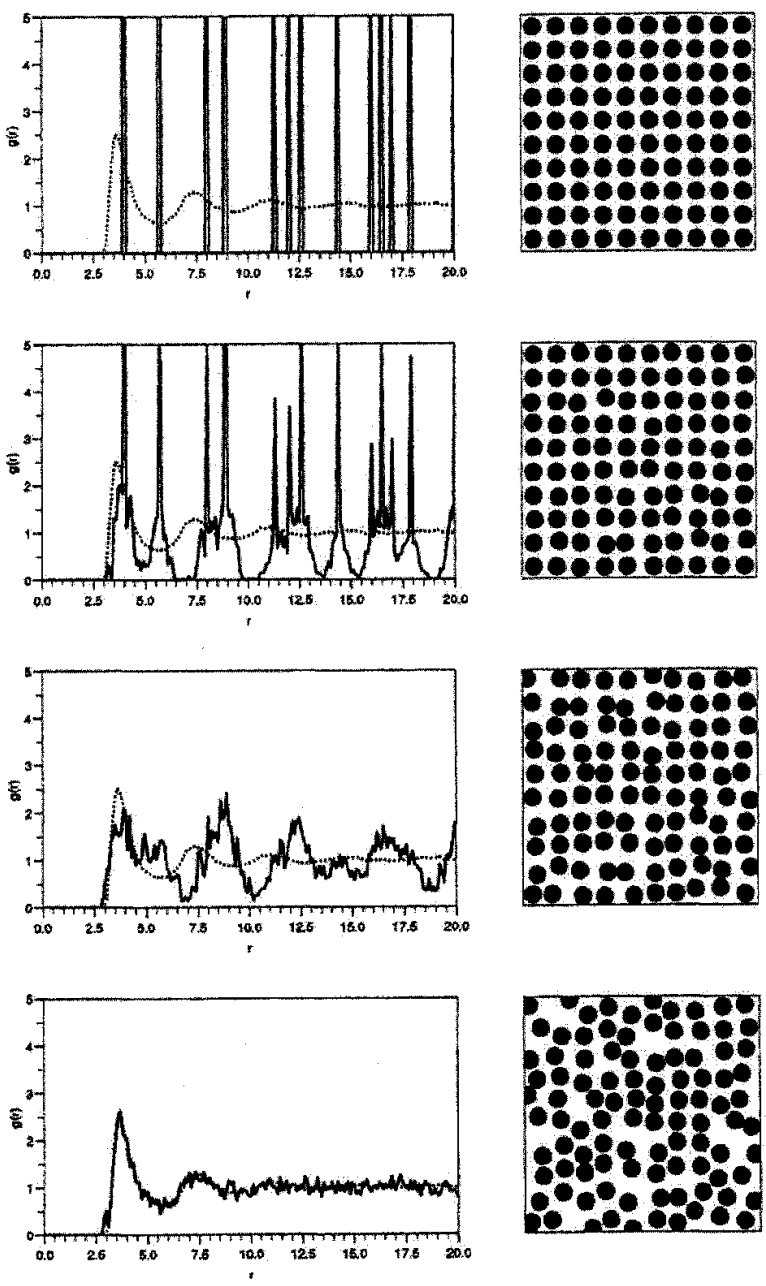

Figure 1. Exemple de modélisation RMC d'un système d'essai simple. L'évolution de la configuration (bi-dimensionelle) est montrée à droite, et à gauche sont comparées la fonction $g^{C}(r)$ calculée (courbe contimue) et la fonction $g^{g}(r)$ expérimentale (courbe pointillée), cette dernière correspondant dans ce cas à une structure obtenue par simulation Metropolis Monte Carlo employant un potentiel Lennard-Jones. La configuration initiale est placée en haut de la figure, suivie par 2 configurations intermédiaires puis la configuration finale en bas. Pour cet exemple, un total de 2500 déplacements atomiques ont été acceptés. Notons le bruit statistique de $g^{C}(r)$ provenant de la petite taille de la configuration. 
L'algorithme RMC s'adapte facilement à l'affinement simultané de plusieurs jeux de données, pourvu que les fonctions représentant ces données (e.g. $F(Q))$ puissent être également calculées à partir de la configuration atomique du modèle RMC. Pour chaque jeu de données un $\chi^{2}$ est défini puis calculé par l'algorithme qui les ajoute simplement pour produire un $\chi^{2}$ total. Les poids relatifs des jeux de données sont donc simplement liés à leurs valeurs différentes de $\sigma$. Les types de données ainsi combinés par le RMC comprennent à présent : la diffraction des neutrons (y compris la substitution isotopique [e.g. 20]), la diffraction des rayons X (y compris la diffusion anomale [e.g. 21]), la diffraction des électrons [8], l'EXAFS [e.g. 22], et le RMN (Magic Angle Spinning [23] et Second Moment [24]). Le nombre le plus important de jeux de données ayant été utilisées est à présent 5 , provenant de 4 techniques expérimentales différentes [24,25]. En pratique la limitation en nombre de jeux de données n'est pas l'algorithme RMC mais plutôt la difficulté expérimentale d'obtenir des jeux de données qui sont cohérents entre eux. Un avantage particulier du RMC est sa tolérance pour des jeux de données incomplets - ils peuvent avoir des gammes de mesure différentes ou bien des résolutions d'appareil différentes, ou même comprendre des "trous" de mesure (e.g. des mauvais points de mesures qui ont été supprimés). Cependant, il est clair que le RMC ne peut pas remplacer une information non-existante dans les données.

\subsection{Contraintes}

Un autre aspect clé du RMC est celui de l'emploi des contraintes. Les contraintes les plus significatives sont la densité et l'utilisation des distances de coupure (les 'cut-offs') qui contraignent l'empilement des atomes et qui font partie intégrante et nécessaire de l'algorithme classique. L'empilement des atomes étant le facteur initial le plus important pour déterminer la structure, il est donc incorporé de façon prioritaire dans l'algorithme. Sans ces deux contraintes (densité et de distance de coupure), le RMC pourrait affiner n'importe quel jeux de données, en dépit d'erreurs expérimentales (systématiques) très larges, pourvu que le nombre de points de données soit moins élevé que le nombre de paramètres affinés (i.e. les $3 N$ coordonnés des $N$ atomes). En introduisant des contraintes, les coordonnées atomiques ne sont plus des variables libres mais sont de fait très couplées. L'importance de ces contraintes est démontrée par le fait que le RMC n'arrive pas à affiner des jeux de données comprenant des erreurs systématiques significatives. En effet, l'incorporation des contraintes de densité et de distances de coupure permet au RMC de signaler et corriger certaines erreurs systématiques simples, telle qu'une mauvaise normalisation d'intensité ou un bruit de fond constant.

Les valeurs choisies pour les distances de coupure ne sont pas toujours évidentes et peuvent être assez importantes lorsque peu de données sont disponibles pour la modélisation. Puisqu'il y a toujours au moins un jeu de données (de diffraction), la plus petite valeur de la distance de coupure peut être directement déduite de la fonction de distribution radiale correspondante. Dans l'absence d'autres informations, cette distance de coupure sera utilisé pour toutes les paires d'atomes. Si plusieurs jeux de données sont disponibles, il est alors possible d'estimer les distances de coupure correspondant aux différentes paires d'atomes. Dans la plupart des cas, il est également possible d'estimer certaines distances de coupure en utilisant d'autres données telles que les rayons atomiques ou ioniques, ou bien des résultats obtenus sur des systèmes voisins, etc... Dans l'absence d'informations détaillées, les distances de coupure doivent être toujours sous-estimées (c'est à dire des contraintes plus faibles) afin de ne pas biaiser l'affinement ce qui reviendrait à fausser la configuration atomique obtenue par le RMC.

La deuxième contrainte généralement utilisée est celle de la coordinence (ou nombre de coordination). Cette contrainte permet en quelque sorte de décrire les liaisons covalentes et joue le rôle d'un potentiel à plusieurs corps. Elle peut être appliquée de plusieurs manières, et peut 
donc être une contrainte forte ou faible. Le nombre de coordination est défini comme le nombre d'atomes de type $\beta$ à une distance comprise entre 2 rayons fixes d'un atome de type $\alpha$. Normalement le rayon inférieur est celui de la distance de coupure entre atomes $\alpha$ et $\beta$. La contrainte est donc que ce nombre de coordination des atomes $\beta$ autour d'un atome $\alpha$ soit égal à un nombre entier choisi. En dénotant $f_{R M C}$ la fraction d'atomes $\alpha$ satisfaisant la contrainte de coordination, et $f_{\text {req }}$ la fraction exigée, le terme

$$
\chi_{c o}^{2}=\left[f_{r e q}-f_{R M C}\right]^{2} / \sigma_{\text {coord }}^{2}
$$

est simplement ajouté au $\chi^{2}$ total. La pondération de la contrainte de coordination est contrôlée par la valeur de $\sigma_{\text {coord }}$. Si cette valeur est très faible (en fait nulle) la contrainte est forte c'est à dire qu'un atome $\alpha$ ayant atteint la coordinence exigée ne le perdra pas. Une valeur plus importante de $\sigma_{\text {coord }}$, i.e. une contrainte faible, favorisera la probabilité qu'un atome $\alpha$ adopte et garde la coordinence choisie. Il est clair que plusieurs contraintes de coordination peuvent être traités en même temps en ajoutant leurs $\chi_{c o}^{2}$ respectifs. Les types d'atomes peuvent être mélangés : par exemple, dans le cas de la silice vitreuse on pourrait contraindre tous les Si à être coordonnés par $4 \mathrm{O}$ et tous les $\mathrm{O}$ par $2 \mathrm{Si}$, tandis que pour le silicium amorphe hydrogéné on pourrait contraindre tous les $\mathrm{H}$ d'être coordonnés par $1 \mathrm{Si}$ et tous les $\mathrm{Si}$ par 4 atomes, ces 4 atomes pouvant être n'importe quelle combinaison de $\mathrm{Si}$ et $\mathrm{H}$.

La coordinence moyenne peut être également contrainte. Cela est rarement employé, mais pourrait être pertinent, par exemple, dans le cas de données EXAFS afin de déduire une coordinence moyenne lorsque une modélisation directe est impossible (e.g. trop de diffusion multiple, fonction de résolution trop compliquée, etc).

Enfin une dernière possibilité est de contraindre la coordinence de certains atomes en particulier plutôt que d'un type d'atomes ( e.g. l'atome 247 reste coordonné à l'atome 591 pendant la modélisation RMC [26]). Il s'agit donc d'une contrainte 'forte' qui fait appel à une liste de de paires d'atomes et de distances interatomiques. Par exemple, dans le $\mathrm{CS}_{2}$ liquide les gammes de distances inter- et intra-moléculaires se recouvrent, empêchant l'emploi d'une contrainte de coordination entre types d'atomes. Par contre, l'emploi d'une contrainte qui coordonne un certain atome $\mathrm{C}$ avec toujours les mêmes deux autres atomes $\mathrm{S}$ garde la molécule intacte en dépit des positions des autres molécules. De la même manière, des unités structurales complexes telles que les polymères peuvent être maintenues lors d'une modélisation RMC.

En principe, la complexité des contraintes mises en œuvre n'est pas limitée. Jusqu'ici seules des contraintes à 2 corps, i.e. appliquées à des paires d'atomes ont été considérées. Les contraintes à 3 corps, telles que celles sur des angles de liaison, peuvent être également employées $[27,28]$. Cependant, il faut bien signaler que de telles contraintes coûtent plus cher en temps de calcul. De la même manière, l'utilisation d'un trop grand nombre de contraintes 'fortes' (comme cela peut être le cas afin de décrire de grandes molécules rigides) peut devenir infaisable car trop chère en temps de calcul.

\subsection{Cohérence des données et des contraintes}

Tout jeu de données expérimentales comprend des erreurs de mesure, qui sont d'ailleurs habituellement sous-estimées. Par conséquent, même si une contrainte est 'sûrement juste', elle n'est pas forcément cohérente avec le jeu de données en question. De la même manière, différents jeux de données peuvent être incohérents les uns avec les autres. Dans de tels cas, on ne peut pas s'attendre à ce qu'une modélisation RMC puisse bien affiner tous les jeux de données tout en respectant les contraintes. Cela peut être lié au modèle lui même qui se coince dans un 'minimum local', mais ce sont plus fréquemment les données et/ou les contraintes sont en sont 
les responsables. Par exemple, la présence de liaisons covalentes assez rigides donne lieu à des oscillations aux grands $Q$ dans le spectre $S(Q)$. Cependant, les effets de diffusion inélastique peuvent déphaser et amortir ces oscillations et on ne sait pas encore corriger ces effets. Par conséquent, une modélisation RMC conduirait à une mauvaise estimation de la coordinence ou bien à un élargissement de la distribution des distances des liaisons covalentes par rapport à celle obtenue par spectroscopie. En revanche, si le modèle RMC est contraint par le bon nombre de coordination ou par la bonne largeur de la distribution de distances de liaison, il n'affinera plus aussi bien les données. C'est l'utilisateur qui décide de donner plus de priorité aux données ou aux contraintes. Par exemple, il est généralement accepté que le Si dans le silice est coordonné par $4 \mathrm{O}$, mais la figure 2 montre qu'un bon affinement des données de diffraction des neutrons et des rayons X n'est obtenu que pour une coordination de 3.75 [29].

Il a déjà été signalé dans ce cours que, lors d'une modélisation RMC, les contraintes sur la densité et sur les distances de coupures entre atomes permettent une correction automatique du bruit de fond (constant) et des erreurs de normalisation des données de diffraction. La présence d'un bruit de fond qui suit une fonction polynomiale de faible degré (erreur systématique assez typique) ne peut pas être affinée par le RMC car elle implique des distances entre atomes trop petites et non physiques. Donc, si la différence entre l'affinement RMC et les données du spectre a la forme d'un polynôme de degré faible, il s'agit d'une bonne indication des erreurs sur les données. Si cette différence n'est pas corrélée avec l'allure du spectre, une correction approximative des données peut s'effectuer en soustrayant un polynôme affiné à cette différence (ceci est aussi possible pour le logiciel MCGR [30]). Par contre, si l'erreur de bruit de fond a une allure liée avec celle du spectre, par exemple le cas d'une diffusion magnétique jusque-là méconnue, la différence (RMC-données) ne sera plus forcément une bonne approximation à l'erreur du bruit de fond et ne devra donc pas servir comme correction aux données.

Ces aspects rendent la modélisation RMC assez utile pour le contrôle de la qualité des données. Même si un utilisateur de RMC se sert du modèle structural le plus simple, et ne le regarde pas, la comparaison entre les données et l'affinement RMC fournit une information rapide sur la qualité des données, et dans le cas de plusieurs jeux de données, sur leur cohérence.

\subsection{L'élaboration des configurations initiales}

Bien qu'en principe le résultat d'une modélisation RMC soit indépendant de la configuration initiale, soumise aux contraintes appliquées; en pratique la convergence de l'affinement RMC est trop lente si la configuration initiale est très loin de la configuration finale - sauf pour les liquides les plus simples. L'élaboration des configurations initiales est donc une étape clé, et une discussion sur les différentes méthodes disponible est nécessaire dans ce cours. Cette discussion se limitera aux méthodes étroitement associées aux logiciels et à la méthodologie RMC standard. Une approche fréquente est d'utiliser le programme RMCA mais sans affiner de données. Cela est alors équivalent à une simulation Monte Carlo de sphères dures (le programme HSMC, ou bien CHSMC si des contraintes de coordinence sont appliquées).

Pour la modélisation de liquides non moléculaires, la méthode la plus simple consiste à créer une configuration aléatoire de points et d'utiliser ensuite le programme HSMC jusqu'à l'obtention d'une configuration d'équilibre ayant des distances de coupure adaptées. 
Pour les liquides moléculaires, les molécules doivent être 'pré-définies' dans la configuration initiale puisque les contraintes de coordinence employées par la suite se suffiront pas à produire $100 \%$ des molécules, mais seulement une fraction importante. Même si la configuration initiale comprend toutes les molécules requises avec des positions et des orientations aléatoires, il y aura certaines distances inter-moléculaires qui seront trop courtes (i.e. plus petites que les

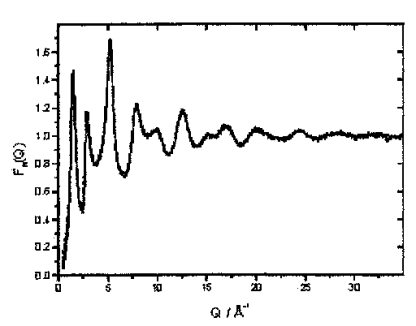

(a)

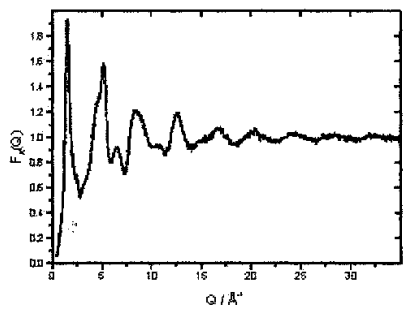

(b)

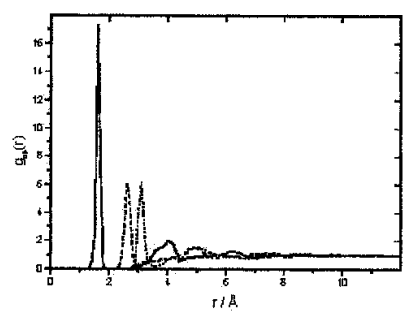

(c)

Figure 2. Facteurs de stincture expérimentaux (courbes continues) pour du silice ( $\mathrm{SiO}_{2}$ vitreux) mesurés par (a) diffraction des neutrons ainsi que (b) diffraction des rayons $\mathrm{X}$ et comparés aux affinements RMC (tirets). En (c) sont représentés les facteurs de structure partiels $g_{\text {Sio }}(r)$ (trait continu), $g_{\circ o}(r)$ (tirets) et $g_{\text {Sisi }}(r)$ (pointille) obtenus du modẻle RMC [29]. 
cut-offs). L'utilisation des contraintes de coordinence entre atomes d'une même molécule dans le programme CHSMC peut garantir la structure moléculaire tout en respectant les distances de coupure. En revanche, les contraintes de coordinence entre types d'atomes sont trop 'faibles' et ne devront donc pas être utilisées. Les polymères fondus sont des liquides moléculaires assez complexes et par conséquent plusieurs méthodes ont été développées pour élaborer la configuration initiale. Les procédures de marche aléatoire (random-walk) qui bâtissent les chaînes monomère par monomère, et reviennent en arrière lorsque les monomères se recouvrent trop, fonctionnent bien pour des monomères simples, mais peuvent se coincer vite lorsqu'il s'agit des chaines longues ayant des groupements latéraux (side-groups) encombrants. D'autre part, des méthodes basées sur des potentiels d'énergie permettent une relaxation des chaînes initialement droites, mais elles ne permettent pas de reproduire toutes les topologies de chaînes existant dans la nature. Une méthode hybride a donc été développée, elle est décrite ci-dessous dans le cas de l'oxyde de poly-propylène [31] :

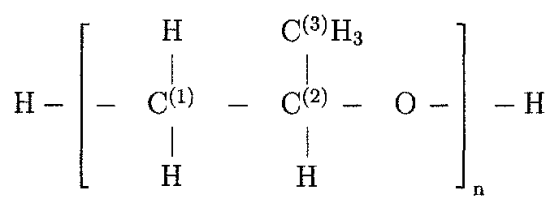

1. Le 'backbone' initial $\left(-\mathrm{C}^{(1)}-\mathrm{C}^{(2)}-\mathrm{O}-\right)_{\mathrm{n}}$ est élaboré par une procédure standard de marche aléatoire. Les dimensions spatiales de la configuration sont déterminées par la densité du polymère complet. Puisque le volume libre est important à ce stade, la procédure est simple et rapide. Les chaines sont terminées de manière appropriée.

2. L'étape suivante consiste à placer 2 atomes $\mathrm{H}$ trop près de l'atome $\mathrm{C}^{(1)}$ (i.e. plus près que le cut-off) et un atome $\mathrm{C}^{(3)}$ plus un atome $\mathrm{H}$ trop près de l'atome $\mathrm{C}^{(2)}$. Le programme CHSMC est exécuté, avec des contraintes adaptées pour maintenir les liaisons chimiques, jusqu'à ce que les distances de coupure soient respectées.

3. Trois atomes $\mathrm{H}$ sont ensuite placés trop près de l'atome $\mathrm{C}^{(3)}$ pour construire un groupement méthyle $\mathrm{CH}_{3}$, puis CHSMC est exécuté à nouveau afin d'équilibrer la configuration en respectant toutes les distances de coupure.

Ce type de procédure hybride est relativement lent mais permet de produire des configurations initiales pour des polymères de n'importe quelle complexité.

En général pour la modélisation RMC des liquides, il n'est pas conseillé d'utiliser une configuration initiale cristalline, même si la densité moyenne est celle du liquid ; cela parce que le cristal initial ne possède souvent pas assez de volume libre pour 'fondre' complètement. Le modèle résultant d'une telle modélisation serait un cristal très désordonné plutôt qu'un liquide, bien que cela ne soit pas trivial en regardant simplement le facteur de structure calculé ou bien la configuration 3-D. Par contre, pour la modélisation des cristaux, il faut bien commencer par la structure cristalline moyenne déterminée par l'une des méthodes cristallographiques standard. Lorsque cela est possible, les paramètres de maille utilisés dans la configuration initiale RMC devraient être obtenus à partir des mêmes données que celles sont modélisées, pour assurer la cohérence.

Les verres de réseau (network glasses) sont les systèmes les plus complexes nécessitant de bonnes configurations initiales. L'emploi de contraintes de coordination implique que la topologie du réseau reste globalement fixe pendant la modélisation ; le RMC ne peut donc affiner les 
coordonnés initiales que d'une manière relativement restreinte. Néanmoins, la méthode RMC a bien réussi la modélisation des verres de réseau, ce qui indique que dans de nombreux cas, la topologie du réseau des liaisons déterminée chimiquement, est la clé de la structure. Pour les verres $\left(\mathrm{K}_{2} \mathrm{O}\right)_{x}\left(\mathrm{SiO}_{2}\right)_{1-x}$ la procédure suivante a été employée [23] :

1. Une configuration aléatoire d'atomes de Si est d'abord créée, avec la densité correspondant au verre final.

2. Le programme CHSMC est exécuté avec des contraintes de coordination sur les voisins ( $\mathrm{Si}$ ). Des données de RMN (magic-angle spinning) permettent de préciser le nombre d'atomes bi, tri ou quadri-coordonnés correspondant au nombre d'atomes de Si possédant 2, 3 ou 4 voisins oxygène pontant (bridging oxygens) nécessaires à la description du modèle final. Les distances initiales Si-Si sont choisies inférieures à 2 fois les distances Si-O permises dans la configuration finale. Si nécessaire, il est également possible d'appliquer une contrainte empêchant la formation d'anneaux de 3-membres [23].

3. Une fois que les contraintes de coordination $\mathrm{Si}-\mathrm{Si}$ sont satisfaites, des atomes d' O sont ajoutés aux centres de toutes les liaisons $\mathrm{Si}-\mathrm{Si}$. Il s'agit des oxygènes pontant.

4. Ensuite des atomes d' $\mathrm{O}$ sont mis près des atomes de $\mathrm{Si}$ ayant moins de $4 \mathrm{O}$ voisins. Ils deviennent donc les oxygènes non pontant. (non-bridging oxygens).

5. Le programme CHSMC est exécuté avec des contraintes de coordination de $4 \mathrm{Si}$ autour de chaque $\mathrm{O}$ et de $2 \mathrm{O}$ autour de chaque $\mathrm{Si}$, jusqu'à ce que tous les cut-offs soient respectés. Si nécessaire, une contrainte sur l'angle de liaison Si-O-Si peut être également appliquée [28].

6. La configuration initiale ainsi obtenue est un réseau silicaté ayant la topologie requise. Les atomes de $\mathrm{K}$ sont ensuite ajoutés de façon aléatoire.

\section{QUELQUES IDEES RECUES}

Jusqu'ici les détails plutôt techniques de la modélisation RMC ont été décrits. Il est également important de prendre un peu de recul en signalant et en discutant certaines des idées reçues et des critiques sur la modélisation RMC. La critique la plus répandue consiste à dire que les modèles obtenus par RMC ne sont pas uniques, ce qui conduit (de façon illogique) à dire qu'ils ne sont pas corrects et donc que la méthode RMC n'est pas utile. Plusieurs remarques doivent être faites ici.

- On ne peut jamais connaître la 'véritable' structure d'un matériau, en ce sens qu'on ne peut pas connaitre les positions exactes de tous les atomes, ou même d'un sous-ensemble d'atomes. Cela s'applique aussi bien pour les matériaux cristallins que pour les noncristallins. La limitation principale est que la structure n'est pas statique mais évolue dans le temps. Dans le plus simple des cas, excepté à $T=0 K$, les atomes vibrent et il y a donc une différence entre la structure moyennée dans le temps et la structure instantanée (celle accessible expérimentalement).

- Un jeu de données expérimentales offre des informations incomplètes sur la structure : par exemple, des corrélations instantanées entre paires d'atomes fournies par la diffraction, ou des projections d'une certaine distribution de densité obtenues par microscopie électronique. 
A partir de ces informations incomplètes le scientifique essaie d'élaborer un modèle permettant une description valable d'un certain aspect de la 'structure'. Le fait qu'on appelle cette procédure 'résolution de la structure' au lieu de 'élaboration d'un modèle' n'implique pas que la 'solution' soit unique ni correcte. Dans ce contexte, la modélisation par RMC n'est pas différente de n'importe quelle méthode de modélisation structurale.

- Un modèle structural obtenu par RMC n'est pas unique. C'est un des avantages de la méthode - et non un inconvenient. Si une méthode ne peut produire qu'un seul modèle (pour un ensemble de données), cela signifie que ce modèle est spécifique à cette méthode ; mais cela ne veut pas dire que le modèle est 'correct' ni qu'il n'existe pas d'autres modèles cohérents avec les mêmes données.

- La modélisation RMC permet d'explorer, e.g. par l'utilisation de différentes contraintes et/ou configurations initiales, une gamme de modèles tous cohérents avec les données disponibles. Bien sûr, certains modèles se ressembleront beaucoup. Cependant, le nombre de ces modèles étant toujours infini (indépendamment du type, de la quantité ou de la qualité des données), l'utilisateur du RMC doit s'imposer certaines limites pour générer ses modèles. Ces différents modèles peuvent être utilisés afin de prédire les résultats de futures expériences, et ils seront ensuite testés. Une telle application du RMC constitue un excellent exemple de la méthodologie scientifique classique !

- Cela nous amène au point clé : le RMC est une méthode de modélisation structurale, rien de plus. Cependant les coordonnées atomiques propres à un certain modèle n'ont pas grande importance. Ce qui compte est l'utilité des modèles. Peuvent-ils aider à la compréhension de certains aspects du matériau, par exemple, la relation entre la structure et une propriété physique?

D'autres aspects peuvent être également discutés :

- Les modèles RMC sont de nature statistique. Ils comprendront toujours, à un certain niveau, des erreurs (des 'défauts structuraux') facilement identifiables mais pas aussi facilement corrigées. En effet, les défauts dans le modèle sont parfois liés aux erreurs provenant des données, et essayer de les corriger ne ferait que produire d'autres défauts. Un petit nombre de défauts ne compromet pas forcément l'utilité d'un modèle.

- Les modèles RMC sont parfois critiqués car il ne prennent pas en compte certaines caractéristiques 'connues'. Mais ce n'est pas la faute de la méthodologie. Les modèles RMC sont fonction des données et des contraintes fournies. Si les caractéristiques manquantes ne résultent pas des données et contraintes fournies, elles sont forcément fondées sur d'autres informations non fournies. Si ces informations étaient fournies, sous forme de données ou de contraintes supplémentaires, ces caractéristiques devraient alors 'apparaître'. De même pour des caractéristiques présentes dans le modèle mais connues comme fausses.

- Cependant, il n'est pas rare que ces caractéristiques 'connues' soient simplement des idées proposées pour expliquer des données précédentes ( $\mathrm{Au}$ fur et à mesure que la bibliographie d'un sujet de recherche se développe, l'expression ' $\mathrm{X}$ a proposé ...' devient ' $\mathrm{X}$ a montré ....' et finalement 'Tl est bien connu que ....'). Si un modèle RMC produit indépendamment une caractéristique 'connue' du système étudié, il s'agit alors d'une bonne confirmation. Sinon, cette caractéristique peut être quand même cohérente avec les données et mise en évidence par l'utilisation d'une contrainte adaptée. Autrement, cela peut s'avérer incohérent avec 
les données. Parfois, des caractéristiques apparemment opposées sont cohérentes avec un unique modèle RMC [33] !

- Si un modèle RMC produit certaines caractéristiques 'inattendues', elles ne devraient pas être vite écartées. Comme toujours, une approche objective vaut mieux qu'une réaction subjective. La modélisation RMC tend à générer la structure la plus désordonnée qui soit cohérente avec les données et les contraintes (bien qu'en pratique la configuration initiale et les paramètres d'execution jouent également un rôle). Il est donc invraisemblable qu'un ordre structural inattendu apparaisse sans être poussé par les données et les contraintes, donc de telles caractéristiques devraient être prises en sérieux. D'autre part, on préconise souvent l'idée du 'rasoir d'Occam' : si une solution simple à un problème peut être trouvée, elle tend à être la bonne, et on devrait donc avoir tendance à écarter des solutions apparemment complexes. Dans le cas des structures désordonnées qui sont poussées par des forces d'entropie, par exemple, il serait risqué d'accepter un modèle manifestant un ordre structural inattendu.

Certaines des idées reçues sont le résultat d'un article théorique publié par Evans [34] au tout début des modélisations RMC. Evans a montré que pour un système pouvant être décrit par un potentiel de paires additif (i.e. des forces entre 2 corps seulement), il existe une relation fonctionnelle unique entre le potentiel et la fonction de corrélation de paires. Le potentiel détermine toutes les fonctions de corrélation de plus grand ordre, et donc la 'structure' complète. La conclusion est donc qu'une connaissance exacte de la fonction de corrélation de paires permet la résolution de la structure. Cette conclusion a été mal interprétée par certains auteurs (mais non par Evans) qui ont suggéré que la modélisation RMC devrait reproduire la 'vraie' structure 3-D, et sinon, la méthode était erronée. Cependant, le résultat d'Evans n'a pas directement de rapport avec la modélisation RMC pour les 4 raisons suivantes :

- La fonction de corrélation de paires (ou le facteur de structure correspondant) doit être connue avec exactitude et sur un domaine infini (en $r$ ou en $Q$ ). Or de vraies données ne sont ni parfaitement exactes et toujours obtenues sur un domaine fini.

- On ne connaît pas la relation fonctionnelle en question ; on sait seulement qu'elle existe.

- Il y a un nombre relativement limité de systèmes pouvant être décrits suffisamment bien par des potentiels de paires additifs.

- La conclusion théorique d'Evans affirme seulement qu'une connaissance exacte de la fonction de distribution de paires détermine le potentiel de paires sans ambiguïté, et par la suite toutes les fonctions de corrélation de plus grand ordre. Pourtant, elle ne traite pas la sensibilité de ces dernières aux inexactitudes présentes dans la fonction de corrélation de paires (ce point a été récemment traité par Soper [35]).

Certes, le résultat d'Evans est indirectement approprié à la modélisation RMC car il suggère qu'un facteur de structure précisément mesuré contient beaucoup d'informations sur les corrélations à 2-corps et à plusieurs corps, et ces informations sont d'autant plus nombreuses que la précision et l'étendue des données augmentent. Cependant, lorsque les contributions à plusieurs corps au potentiel sont importantes, les données à 2-corps (i.e. la fonction de corrélation de paires) sont vraisemblablement insuffisantes, d'où l'introduction, par exemple, de contraintes de coordination dans le RMC. 
Plusieurs études d'essai de RMC ont eu pour but l'investigation de la dérivation des corrém lations de maint-corps à partir de la fonction de corrélation de paires ou du facteur de structure calculés d'un modèle 3-dimensionnel original. Howe et McGreevy [36] ont trouvé, pour un système mono-atomique, que les corrélations à 3-corps simples sont bien reproduites, même si les fluctuations statistiques des corrélations à 2-corps et à 3-corps ont tendance à être supprimées. Pour un système de molécules diatomiques, i.e. où les forces à plusieurs corps sont significatives et donc à l'abri de la théorème d'Evans, la modélisation RMC sans contraintes ne marche pas. Elle produit un mélange de coordinations atomiques bien que la coordination désirée (diatomique) domine. Par contre, l'application d'une contrainte de coordination adaptée aux molécules diatomiques a permis l'obtention de résultats raisonnables. Colognesi et al. [37] ont examiné la question des corrélations orientationnelles entre molécules diatomiques simples mais en ont conclu que le RMC ne les reproduisait pas très bien. Cependant, Soper [35] a mené une série de tests qui montrent qu'un grand nombre de potentiels à 2-corps peuvent être générés avec la méthode EPSR (voir la section 5.3), pour les systèmes atomiques ainsi que moléculaires, et que ces potentiels reproduisent bien les corrélations à 3-corps initiales. Les conclusions de Howe et McGreevy [36] sont ainsi soutenues par Soper [35].

Les conclusions différentes de Colognesi et al. [37] sont certainement liées à la méthode de 'contrôle de qualité' utilisée. Cinq fonctions de corrélations orientationnelles ont été calculées, mais les fluctuations caractéristiques n'ont pas été déterminées. Par exemple, par combien varieraient ces fonctions parmi un ensemble de configurations initiales, ou en fonction des petites variations dans les limites angulaires employées? - c'est à dire, est-ce que ces fonctions de corrélations orientationnelles sont assez robustes pour pouvoir évaluer les différences entre le modèle original et celui reproduit par RMC ? Soper [35] a employé une seule représentation générale des corrélations angulaires, illustrée dans la figure 3 , permettant un 'contrôle de qualité' visuel.

Même si ce genre d'étude test est intéressant, il faut quand même souligner que les résultats d'une modélisation de 'vraies' données, soit par RMC soit par une autre méthode, doivent toujours être interprétés avec prudence et un esprit ouvert. Si l'utilisateur de RMC s'intéresse en détail à certaines corrélations à plusieurs-corps, il devra employer des contraintes adaptées afin explorer l'ensemble des corrélations cohérentes avec les données.

\section{VARIATIONS SUR LA METHODE RMC CLASSIQUE}

\subsection{Molécules rigides}

Dans le cas des molécules relativement rigides, il n'est pas forcément utile de déplacer des atomes individuellement comme dans l'algorithme RMC classique et un programme RMC spécial (RMCMOL) a été développé. Dans ce programme, les déplacements consistent en des translations et rotations de groupes atomiques pré-définis, i.e. des molécules [38,39]. Pourtant, en pratique, ce programme ne s'est pas avéré être plus efficace que les déplacements atomiques individuels. En effet, à cause des fortes contraintes stériques, seuls les déplacements de petites molécules étaient acceptés avec une probabilité élevée. De plus, il y avait des inconvénients :

- La molécule doit être pré-définie. Les informations pertinentes sont normalement obtenues par l'affinement de la partie grands $Q$ du facteur de structure pour en extraire le facteur de forme intra-moléculaire. Ce facteur de forme est en fait plus important à petits $Q$, mais dans cette région il concide avec les corrélations inter-moléculaires. Toute erreur dans l'affinement du facteur de forme sera ainsi amplifiée à petits $Q$ et engendrera donc 


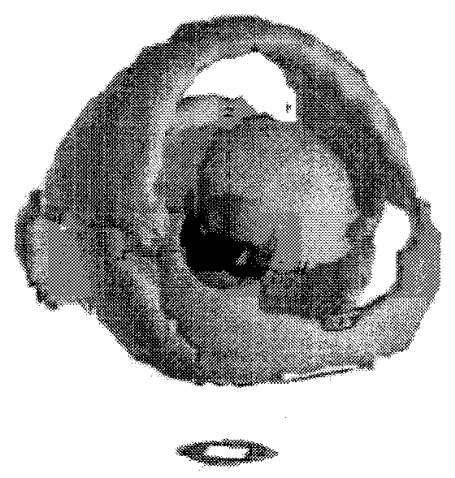

Figure 3. La fonction de densité spatiale de leau déterminee par nodelisation EOSR [35]. An

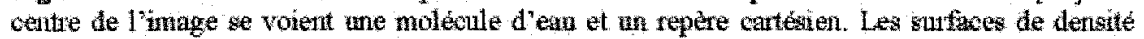
autour de cette molecule centrale correspondent a la denatié relative de nolécules d'ean en fonction des coctonnes polaires $(r, \theta, \phi)$ lies an reperte cartesien. L'isomstrface nontude correspond à une densité de 1.35 x la densite de lean en état massif.

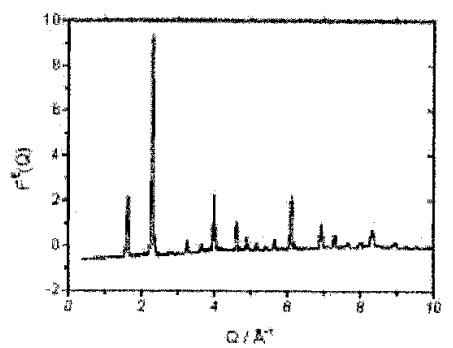

(a)

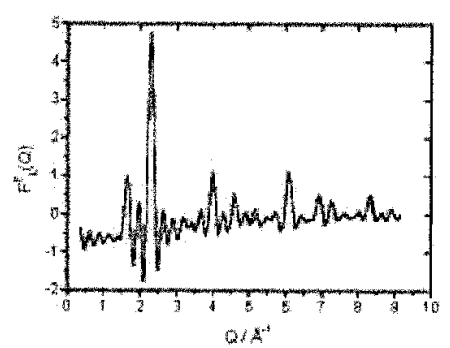

(b)

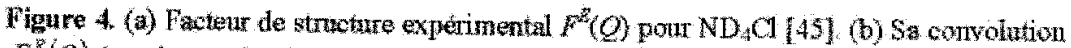
$F_{i}^{R}(Q)$ (combe contine) et son affinement $\mathrm{RMC}$ (brets) 
une erreur dans le facteur de structure inter-moléculaire qui est la fonction affinée par l'algorithme de RMCMOL. Ce problème est bien illustré par les résultats sur le phosphore liquide où une approche en molécules rigides n'a pas pu bien affinée les données [40], tandis qu'une approche de déplacements atomiques avec contraintes de coordination a bien réussi [41].

- Lors du calcul du facteur de structure (qui est comparé aux données), RMCMOL tient compte des vibrations intra-moléculaires en introduisant un facteur de Debye-Waller dans le facteur de forme. Pourtant, le modèle comprend seulement des molécules rigides, possédant donc d'un volume libre plus important que celui dans la réalité. Soper [35] a également donné cette raison afin d'expliquer le désaccord entre les fonctions de distribution de paires expérimentales et les simulations sur l'eau (de Monte Carlo ou de Dynamique Moléculaire) employant des molécules rigides. Évidemment, plus les molécules sont flexibles, moins l'approche de molécules rigides est applicable.

\subsection{La méthode de convolution pour les matériaux de poudre cristallins}

Dans la section 2.1, nous avons expliqué que l'algorithme RMC classique essayait d'affiner soit le facteur de structure expérimental $F^{E}(Q)$, soit la fonction de distribution radiale (de paires) $g^{E}(r)$. Alors, soit $F^{C}(Q)$ est obtenu par transformation Fourier de $g^{C}(r)$, soit $g^{E}(r)$ doit être obtenu par transformation Fourier de $F^{E}(Q)$. Dans les deux cas, il faut éviter des erreurs provenant de la troncation de la fonction qui est transformée. Pour obtenir un bon $g^{E}(r)$ il faut donc mesurer $F^{E}(Q)$ jusqu'à une grande valeur de $Q$, ou bien utiliser une méthode inverse (e.g. MCGOFR [42] ou MCGR [30]). Pour obtenir un bon $F^{C}(Q)$ il faut employer une configuration RMC assez grande pour s'assurer que $g^{C}(r)$ est plat à la valeur calculée de $r$ la plus grande (la moitié d'une arête, dans le cas d'une configuration RMC de géométrie cubique). Pour la plupart des liquides/verres, cette dernière condition est satisfaite par des modèles de quelques milliers d'atomes. Par contre, les matériaux cristallins, qui possèdent un ordre à grande distance, auraient besoin de quelques milliards d'atomes. Deux approches sont alors proposées : soit $g^{E}(r)$ est affiné par l'algorithme RMC, soit $F^{E}(Q)$ est affiné mais l'effet de troncation en $r$ (dû aux dimensions finies de la configuration RMC) est pris en compte par la convolution de $F^{E}(Q)$ avec la transformation Fourier de la fonction de troncation :

$$
\begin{array}{ll}
m(r)=1 & r<L / 2 \\
m(r)=0 & r>L / 2
\end{array}
$$

Mellergård [44] a signalé que les équations originalement proposées par Nield et al. [43] ne sont pas tout à fait justes, et que la fonction devant être convoluée est effectivement $Q \cdot F^{E}(Q)$, c'est à dire :

$$
F_{L}^{E}\left(Q_{j}\right)=\frac{1}{\pi Q_{j}} \int Q_{i} F^{E}\left(Q_{i}\right)\left[\frac{\sin \left(\left|Q_{i}-Q_{j}\right| L / 2\right)}{\left|Q_{i}-Q_{j}\right|}-\frac{\sin \left(\left|Q_{i}+Q_{j}\right| L / 2\right)}{\left|Q_{i}+Q_{j}\right|}\right] d Q_{i}
$$

La figure 4 montre, pour le système $\mathrm{ND}_{4} \mathrm{Cl}$, un exemple de données $F^{E}(Q)$ convoluées et l'affinement RMC correspondant. L'erreur dans les équations explique que les oscillations de l'affinement à petit $Q$ soient plus importantes que celles des données $F^{E}(Q)$ convoluées. La figure montre également que cette méthode de convolution fait perdre forcément certaines informations concernant l'ordre à grande distance :l'élargissement des pics qui en résulte a tendance à supprimer les pics de Bragg faibles, surtout lorsqu'ils se trouvent à coté des grands pics. Pour 
cette raison, une nouvelle méthode a été développée pour les matériaux cristallins. Elle est décrite dans la prochaine section.

\subsection{Méthode de calcul direct pour les matériaux de poudre cristallins}

La méthode de calcul direct pour les matériaux de poudre cristallins fait partie du logiciel RMCPOW et a déjà été décrite en détail ailleurs $[12,13,46,47]$. Les dimensions de la configuration RMC sont choisies comme des multiples de celles de la maille cristalline, c'est à dire une supercellule, et le facteur de structure total $F(Q)$ est calculé en utilisant l'expression :

$$
F(\mathbf{Q})=\frac{1}{N}\left\langle\sum_{j, j^{\prime}=1}^{N} e^{-i \mathbf{Q} \cdot\left[\mathbf{R}_{j}(0)-\mathbf{R}_{j^{\prime}}(0)\right]}\right\rangle=\frac{1}{N}\left\langle\left|\sum_{j=1}^{N} e^{-i \mathbf{Q} \cdot \mathbf{R}_{j}(0)}\right|^{2}\right\rangle
$$

où la somme est sur les $N$ atomes dans l'échantillon ayant les positions $\mathbf{R}_{j}(t=0)$ c'est à dire les atomes dans la boîte de configuration. Ici on néglige les longueurs de diffusion des atomes, et les brackets \langle\rangle indiquent une moyenne angulaire sur toutes les orientations de la configuration. La fonction $F(Q)$ représente alors l'intensité diffusée totale comprenant la diffraction de Bragg et la diffusion diffuse (i.e. déjà intégrée sur les échanges d'énergie entre faisceau et échantillon et correspondant donc à une image instantanée de la structure). Pourtant, dans la cristallographie conventionnelle, seulement la diffraction de Bragg élastique est considérée, elle correspond au facteur de structure élastique définit par :

$$
S(\mathbf{Q})=\frac{1}{N}\left\langle\sum_{j, j^{\prime}=1}^{N} \int e^{-i \mathbf{Q} \cdot\left[\mathbf{R}_{j}(0)-\mathbf{R}_{j^{\prime}}(t)\right]} d t\right\rangle
$$

En première approximation (i.e. en supposant des vibrations harmoniques de faible amplitude), l'intégration peut être effectuée et la somme sur les $N$ atomes dans l'échantillon (i.e. la configuration RMC) peut être remplacée par une somme sur les $N_{c}$ atomes dans la maille élémentaire, donnant l'expression plus familière:

$$
S(\mathbf{Q})=\frac{1}{N_{c}} e^{-W Q^{2}}\left\langle\left|\sum_{k=1}^{N_{c}} e^{-i \mathbf{Q} \cdot\left\langle\mathbf{R}_{k}(t)\right\rangle}\right|^{2}\right\rangle
$$

où les $\left\langle\mathbf{R}_{k}(t)\right\rangle$ sont les positions moyennées en temps des atomes dans la maille élémentaire et $W$ est le facteur de Debye-Waller (DWF) lié au déplacement moyen au carré des atomes par rapport à leur position moyenne.

La fonction $S(Q)$ est donc déterminée par les corrélations entre les positions moyennes des atomes (l'équation 11), tandis que $F(Q)$ est déterminée par les corrélations entre les positions instantanées (l'équation 9). De façon plus simple, le facteur de structure élastique donne une image 'moyennée dans le temps' de la structure du matériau, tandis que le facteur de structure total en donne un 'cliché'. Les deux images ne sont égales qu'à une température de $T=0 \mathrm{~K}$. $\grave{A}$ d'autres $T$ la structure n'est pas statique mais dynamique et donc ni l'une ni l'autre des images n'est 'juste'. L'image la plus utile dépend du problème étudié. La plupart des études cristallographiques s'intéressent à la structure moyennée dans le temps et donc la diffraction élastique suffit (d'où la soustraction habituelle à un spectre avant l'affinement d'un 'bruit de fond', comprenant en fait la diffraction diffuse et la plupart de la diffraction inélastique. Pour un liquide, par contre, tous les atomes sont libres de diffuser à grande distance et il n'y a donc pas de structure moyennée en temps et par conséquent pas d'intensité strictement élastique - seulement l'image instantanée de la structure a alors du sens. Pour les structures cristallines manifestant 
un désordre dynamique, par exemple les conducteurs d'ions rapides, les 2 images (moyennée en temps et instantanée) peuvent être valables.

Bien qu'il soit possible de modéliser par RMC la structure moyenne des matériaux cristallins, basée sur le facteur de structure élastique $S(Q)$, cela n'a été jamais été fait excepté pour tests simples. Il y a de nombreuses autres méthodes, plus efficaces que le RMC, pour modéliser/affiner les structures cristallines moyennes (e.g. le Rietveld). Cependant il convient de signaler certaines méthodes de Monte Carlo qui sont conceptuellement très proches du RMC [e.g. 48]. D'autre part, le RMC permettant de modéliser la structure instantanée à partir du facteur de structure total, il fournit donc des informations sur les corrélations atomiques locales dans les cristaux, ce qui n'est pas possible avec les techniques classiques d'affinement cristallographique.

Puisque qu'une configuration RMC possède des conditions aux limites périodiques, la fonction $F(Q)$ calculée via l'équation 9 représente en fait la diffraction de Bragg provenant de la super-cellule (comprennant plusieurs mailles cristallines). Cette diffraction comprend beaucoup de pics à cause de la grande taille de la super-cellule, les pics correspondant à la maille élémentaire n'en sont qu'un petit sous-ensemble. S'il n'y a pas de déplacements atomiques, tous les pics de la super-cellule qui ne sont pas des pics de la maille élémentaire ont une intensité nulle. Lorsqu'il y a de petits déplacements atomiques, tous les pics peuvent avoir une intensité non-nulle, mais les pics de la maille élémentaire ont tendance à être les plus intenses. Au fur et à mesure que la taille de la configuration (super-cellule) augmente, les pics additionnels de la super-cellule fusionnent pour former un bruit de fond : la diffusion diffuse. Ainsi, si nous parvenons à élaborer une configuration RMC assez volumineuse, le calcul direct par l'équation 9 donnera une estimation raisonnable aussi bien de la diffraction de Bragg que de la diffusion diffuse. Puisque le diffractogramme RMC est calculé directement dans l'espace $Q$, il peut être facilement convolué par la fonction de résolution expérimentale. Si l'échantillon est magnétique, une modélisation de la structure magnétique peut s'effectuer en même temps, la diffraction magnétique étant calculée selon des équations similaires [13]. En pratique, pour une configuration RMC de taille raisonnable, disons 4000 atomes, les pics de la super-cellule sont suffisamment nombreux mais ils ne sont pas uniformément distribués en $Q$. Aux petites valeurs de $Q$, où ces pics sont relativement rares, il est nécessaire de les lisser ensembles pour produire une bonne estimation de la diffusion diffuse $[13,46]$. Un avantage particulier de RMCPOW est que les résultats pour la structure moyenne peuvent être comparés directement à ceux d'un affinement Rietveld basé sur les mêmes données. Pour des cristaux très ordonnés, les 2 résultats devraient être très semblables ; pour des cristaux désordonnés, on s'attend à voir des différences car l'affinement Rietveld n'a pas besoin d'affiner en même temps la diffusion diffuse, seulement les pics Bragg (c'est à dire, la diffraction élastique). De l'autre coté, l'affinement par Rietveld est beaucoup, beaucoup plus rapide que le RMC !

Tucker et al. ont développé une méthode hybride pour la modélisation des facteurs de structure totaux $[49,50]$. Leur méthode permet l'affinement simultané de $g(r), F(Q)$ (calculé via transformation Fourier) et des intensités élastiques de Bragg (calculées via l'équation 11). Bien que ces 3 informations proviennent d'un même jeu de données, le modèle structural qui résulte de ce triple-affinement dépendra un peu des pondérations relatives - $g(r)$ mettant l'accent sur l'ordre à courte distance et les intensités élastiques de Bragg sur l'ordre à longue distance.

\subsection{Méthode de calcul direct pour les matériaux monocristallins}

Alors qu'on pourrait penser que les données de diffraction d'un monocristal sont préférables à celle d'une poudre puisque qu'elles donnent des informations 3-dimensionnelles plutôt qu'une moyenne uni-dimensionnelle; nous allons voir qu'en pratique, il y a quelques difficultés. 
- Les données de diffraction sur monocristaux sont obtenues pour un plus petit nombre de point en $Q$ (i.e. dans l'espace réciproque) et pour un nombre restreint de plans de symétrie.

- Elles sont plus difficilement corrigées et normalisées à une échelle absolue.

- La résolution instrumentale modifie différemment les intensités de la diffraction de Bragg et de la diffusion diffuse.

- Il est plus difficile de produire des échantillons convenables.

- Pour la diffraction des neutrons, il est difficile d'effectuer l'intégration sur l'énergie à travers toute la gamme en $Q$. Par conséquent, l'intersection (dépendant de la longueur d'onde incidente) du chemin d'intégration avec la courbe de dispersion des phonons peut faire apparaître des 'formes' caractéristiques dans l'intensité de diffusion diffuse.

La méthode de calcul direct pour les données de diffraction de neutrons sur monocristaux fait partie du programme RMCX [11], mais à présent il n'a servi que pour modéliser les données de diffusion diffuse de neutrons et non de diffraction de Bragg. De plus, le programme DISCUS, qui calcule l'intensité de diffraction diffuse, comprend maintenant un sous-programme d'affinement RMC qui a déjà été appliqué aux données de diffraction neutrons et rayons $\mathrm{X}$ [51-53].

\section{METHODES CONNEXES}

Avant de discuter d'autres méthodes de modélisation structurale qui sont semblables au RMC, il faut souligner encore un point : n'importe quelle méthode produisant un modèle cohérent avec les données est aussi valable que le RMC, éventuellement plus valable si elle prend en compte des contraintes thermodynamiques (ce que le RMC ne fait pas). Il est rare que les modèles structuraux non basés sur des données puisse être cohérent avec celles-ci aux erreurs expérimentales près, en revanche il est fréquent qu'un 'bon accord' soit revendiqué dans ce caslà. Un même niveau d'accord entre les données et un modèle RMC serait sûrement critiqué et qualifié d'insuffisant et problématique! On devrait toujours se rappeler qu'un modèle de sphères dures adéquat donne presque toujours une première approximation juste d'une structure, et donc la réussite d'un modèle quelconque devrait être jugée par rapport à ce résultat. D'autre part, on devrait également se rappeler qu'il n'existe pas de relation linéaire entre 'qualité de l'affinement' et 'qualité du modèle'.

\subsection{D'autres variations}

Comme discuté ci-dessus, la méthode d'échantillonnage statistique employée en RMC n'a pas de signification particulière ; elle est simplement un moyen pour obtenir des modèles cohérents avec les données. Pourtant, certains auteurs ont essayé 'd'améliorer' le RMC en modifiant la méthode d'échantillonnage statistique. Toth et Baranyai ont mené une série de tests détaillés, par exemple en contraignant l'entropie lors de l'échantillonnage, ce qui a rendu l'algorithme plus efficace [54-56]. De telles modifications peuvent être utiles, en particulier pour les configurations de grande taille, mais il faut énoncer certains points pertinents :

- L'algorithme RMC 'classique' est plutôt inefficace, mais il s'agit d'une politique délibérée afin de le rendre le plus général et facilement modifiable. 
- De nouvelles méthodes d'échantillonnage pourraient être plus justes en termes statistiques dans le cas de données idéales. Pourtant, le plus grand problème des vraies données sont les erreurs systématiques, qui peuvent rendre des méthodes d'échantillonnage plus sophistiquées inappropriées. Ces nouveaux algorithmes devraient donc être testés sur de vraies données.

- L'addition de telles contraintes 'théoriques' (e.g. impliquant l'entropie) risque de donner l'impression que le RMC essaie de produire la 'vraie' structure, alors que nous avons a déjà souligné que cela n'était pas son but.

da Silva et al. ont employé une méthode où la fonction de distribution radiale est calculée à partir d'un ensemble de configurations générées consécutivement [57-60]. Ces configurations sont donc corrélées et pour cette raison la méthode n'est équivalente ni à celle impliquant une seule configuration de taille plus grande ni à celle de plusieurs configurations indépendantes. De plus, ces auteurs utilisent une valeur de $\sigma$ très petite, ce qui peut donner lieu à un accord quasiment parfait avec les données (pourvu que la configuration initiale soit suffisamment proche du résultat final). Ceci est revendiqué comme un avantage de la méthode. Bien que cela soit passible dans le cas de données idéales, pour de vraies données, il est rare qu'un accord parfait soit possible, voire raisonnable. Une autre revendication est de dire que ce nouvel algorithme ne nécessite pas l'utilisation de contraintes ' $a d h o c$ ' telles que les distances de coupure (distances minimales entre atomes). Cependant, l'utilisation d'une contrainte sur la distance de coupure est complètement équivalente à l'utilisation d'un jeu de données ayant $g\left(r<r_{o}\right)=0$ et $\sigma\left(r<r_{o}\right) \approx 0$, à cet égard, leur méthode est donc identique au RMC 'classique' dont la formulation en termes de distance de coupure est simplement plus transparente et compréhensible physiquement.

Adams et Swenson $[61,62]$ ont ajouté au programme RMCA la possibilité de contraindre la somme des valences des liaisons (i.e. le nombre total d'électrons partagés dans les liaisons covalentes) calculée à partir d'une configuration. Cela est similaire à l'inclusion d'un potentiel de forme simple. Si cette contrainte est relativement douce, elle a essentiellement l'effet d'empêcher certains défauts structuraux qui pourraient être produits lors de l'échantillonnage statistique. De plus, la valence des liaisons des modèles finaux peut également être utilisée de façon auto-cohérente pour calculer, par exemple, les chemins de conduction ionique. Une contrainte semblable est maintenant disponible dans le programme RMCPOW [63].

\subsection{La simulation par Metropolis Monte Carlo (MMC) et d'autres méthodes de Monte Carlo}

La méthode la plus semblable au RMC est celle de la simulation par Metropolis Monte Carlo (MMC). Tandis que le RMC requiert des données, le MMC requiert un potentiel interatomique. Les résultats de MMC sont donc thermodynamiquement cohérents mais pas forcément en accord avec les données, et inversement pour le RMC. En général, le MMC n'est pas souvent employé pour élaborer des modèles structuraux de 'vrais systèmes', mais assez souvent pour produire des modèles statistiques de grande taille pour des 'systèmes idéaux' (e.g. les alliages substitutionnels ou les modèles Ising magnétiques). Le MMC est également employé dans plusieurs méthodes d'affinement de potentiel, cela sera traité dans la prochaine section.

Deux autres méthodes de Monte Carlo méritent d'être signalées car elles sont souvent confondues avec le RMC. La méthode de Monte Carlo Inverse $[18,19]$ est une méthode très particulière permettant d'obtenir les énergies d'interaction effectives dans le cas des alliages binaires. Elle est basée sur des modèles structuraux eux-mêmes déterminés par la méthode de Monte Carlo de Gehlen et Cohen [64] qui utilise des paramètres d'ordre à courte distance provenant de données 
de diffusion diffuse. Le terme 'recuit simulé' (simulated annealing) fait référence à une méthode de Monte Carlo générale pour atteindre le minimum global d'une fonction de configuration [65]. Par exemple, le minimum global de l'énergie du système simulé (i.e. son vrai état fondamental) peut être le but d'une simulation MMC. Ceci est accompli par réduction progressive d'une variable de contrôle (ici la température) à partir d'une valeur élevée, d'où le mot descriptif de 'recuit'. Bien qu'un recuit simulé soit parfois utilisé dans le RMC pour éviter un minimum local avec une valeur trop élevée de $\chi^{2}$, il faut souligner à nouveau que le RMC ne cherche pas un minimum global en $\chi^{2}$. N'importe quelle configuration ayant une valeur de $\chi^{2}$ suffisamment faible est une configuration valable. Il est même possible que la configuration de $\chi^{2}$ minimal soit pire puisqu'elle est affinée aux détails des erreurs (systématiques) des données.

\subsection{Méthodes d'affinement d'un potentiel}

Plusieurs méthodes produisant un modèle structural à partir des données expérimentales, mais via l'affinement d'un potentiel interatomique [e.g. 66-71], ont été développées. La plus utilisée est sans doute la méthode EPSR (Empirical Potential Structure Refinement) développée par Soper [69]. A première vue, il semblerait que de telles méthodes produisent des résultats plus justes que ceux du RMC puisqu'ils sont cohérents avec les données et un potentiel. Pourtant, quelques precautions sont à prendre.

Les méthodes d'affinement d'un potentiel utilisent toutes le même genre d'algorithme. Tout d'abord, un potentiel initial est utilisé pour générer une structure initiale (e.g. via MMC) et pour calculer un facteur de structure (ou une fonction de distribution radiale). Le résultat est alors comparé aux données expérimentales. La différence entre le facteur de structure (ou la fonction de distribution radiale) calculé et les données est ensuite utilisée pour générer un nouveau potentiel, et ainsi de suite. Lorsque l'accord entre calcul et données satisfait certaines conditions, le potentiel peut être alors employé pour générer plusieurs configurations (toutes cohérentes avec les données) qui permettent de calculer, par exemple, des fonctions de corrélation de plus grand ordre avec une précision statistique élevée (voir la figure 3). L'échantillonnage statistique et la cohérence thermodynamique sont certainement meilleurs que ceux du RMC, et l'emploi d'un potentiel évite la production de certains défauts dans le modèle. Néanmoins, il faut se poser quelques questions :

- Est-ce que le modèle structural généré est influencé par le choix arbitraire de la forme du potentiel? Qu'est-ce qui arrive si cette forme n'est pas bien adaptée au système modélisé? Dans de nombreux cas, lorsque le système peut être décrit par un potentiel à 2-corps effectif, cela ne pose probablement pas de problème. En revanche, lorsque les effets des forces à plusieurs-corps sont significatifs (ceci n'impliquant pas forcément que ces forces ellesmêmes soient importantes), ce choix arbitraire du potentiel risque d'empêcher certaines configurations atomiques. Certes, il n'y a aucune garantie pour que le RMC fasse mieux. Pour les systèmes proches d'une transition (e.g. de métal-isolant), le fait d'imposer une forme simple et globale du potentiel peut générer de mauvais résultats (de même pour n'importe quelle simulation employant un potentiel simple et global).

- Est-ce que le potentiel affiné est valable pour autre chose que la production d'un modèle structural, par exemple, est-ce qu'il peut servir dans une simulation de dynamique moléculaire? Probablement pas... Dans un cas simple comme la modélisation de l'argon liquide basée sur un potentiel de Lennard-Jones, où l'on sait que la forme du potentiel est correcte et qu'il n'y a que 2 paramètres à déterminer, il serait étonnant qu'il y ait un problème avec le potentiel affiné. Cependant Soper [35] a mené une série de tests qui montrent que 
plusieurs potentiels peuvent être affinés par EPSR, aussi bien pour des systèmes atomiques que moléculaires, qui reproduisent bien les facteurs de structure expérimentaux et même les corrélations à 3-corps - c'est à dire, les différents potentiels correspondent tous à une bonne représentation de la structure. Ces résultats suggèrent que les potentiels affinés, au moins ceux qui sont relatifs aux méthodes d'affinement développées jusqu'ici, sont utiles dans le contexte du programme de modélisation, mais ne devraient pas être utilisés hors de ce contexte-là.

Rappelons que si une forme et des paramètres initiaux adaptés sont choisis pour le potentiel, le facteur de structure résultant de la modélisation peut être en bon accord avec les données même si elles contiennent d'importantes erreurs [e.g. 27]. De mauvaises données ou des données limitées ne contiennent que des informations limitées, et aucune méthode ne peut en extraire davantage d'informations. Notons également que certains types d'erreurs dans les données mènent à des résultats prévisibles dans la modélisation RMC : par exemple, une pente dans le bruit de fond à grand $Q$ cause souvent un pic aigu ou une marche accentuée à petit $r$ dans le $g(r)$. Le fait d'imposer un potentiel dans la modélisation étalera ces effets d'une manière moins prévisible.

En tenant compte de ces avertissements, il est clair que, pour la modélisation structurale de beaucoup de liquides, une méthode telle que celle de l'EPSR a suffisamment d'avantages sur le RMC pour être conseillée. Pour la modélisation des verres, cela n'est pas encore évident, bien que les verres métalliques soient certainement bien adaptés à une telle méthode car il s'agit quasiment des systèmes de sphères dures. Il n'est pas encore clair que la méthode soit valable pour les matériaux cristallins et nous pouvons résumer ainsi : le RMC est une méthode plus générale et peut donc s'appliquer à presque n'importe quel système, tandis que l'EPSR est plus spécifique et fournira de meilleurs résultats dans les cas appropriés.

\subsection{Simulations de dynamique moléculaire}

La simulation par dynamique moléculaire (DM) classique requiert un potentiel interatomique en entrée. Comme le MMC, la dynamique moléculaire est thermodynamiquement cohérente, et possède l'avantage évident et important qu'à la fois la structure et la dynamique du système sont simulées. Si la simulation en dynamique moléculaire reproduit les données expérimentales, elle est alors sans aucun doute la méthode la plus appropriée. S'il y a des désaccords, la simulation n'est pas forcément inutilisable - tout dépend du niveau de désaccord et du but de la simulation. Des informations ou des données de dynamique (e.g. des constantes de diffusion, des courbes de dispersions des phonons ou bien la densité d'états vibrationnelle) peuvent aussi servir pour tester une simulation DM.

Les méthodes de dynamique moléculaire ab-initio, comme la méthode de Car-Parrinello [73], sont en principe indépendantes d'un potentiel car elles accordent aux électrons un traitement complet de la mécanique quantique. En pratique seulement les électrons de valence sont traités de cette manière et des pseudo-potentiels sont employés pour décrire les électrons de coeur. La méthode coûte très cher en temps de calcul et par conséquent la taille de la configuration simulée est typiquement limitée à environ 100 atomes maximum et aux temps de simulation de quelques picosecondes. Les effets de taille peuvent être donc significatifs, bien que certaines techniques d'ab-initio aient été développées afin de permettre une comparaison plus réaliste avec des données expérimentales structurales et dynamiques [74]. Certaines simulations ont montré un accord raisonnable avec l'expérience mais d'autres ont révélé un désaccord sérieux. Malgré cela, un avantage majeur des simulations ab-initio est l'accès aux informations concernant la distribution de la densité électronique de valence (i.e. les liaisons), particulièrement dans les cas où il y a des fluctuations locales significatives. 
En général, les méthodes de simulation MD et RMC peuvent être considérées comme complémentaires - elles abordent parfois le même problème mais par des moyens différents. Une approche récente consiste à employer le RMC pour aider affiner des potentiels utilisés dans les simulations MD [e.g. 75].

\section{CONCLUSIONS}

La modélisation par RMC est maintenant une technique bien établie qui s'est montrée capable de contribuer à la compréhension des structures d'une gamme très large de matériaux. Le RMC offre des possibilités uniques pour la combinaison quantitative d'une gamme de différents types de données expérimentales, par exemple pour les études d'ordre local magnétique, et il reste encore beaucoup de perspectives pour son développement futur. Il semblerait donc que la méthode de $\mathrm{RMC}$ soit encore là pour longtemps !

À part les aspects détaillés qui ont été discutés dans ce cours, il y a 3 points généraux qui doivent être retenus. Premièrement, des données exactes et quantitatives sont disponibles, sans tenir compte de leur allure : des pics fins dans le cas de diffraction des cristaux, ou bien des oscillations larges dans le cas de diffraction ou de l'EXAFS des liquides. Deuxièmement, la procédure d'élaboration des modèles structuraux véritables (i.e. atomistiques) apporte des informations considérables, souvent sans tenir compte de la méthode de modélisation utilisée, ou sans que les modèles soint cohérents avec les données. Troisièmement, il n'y a rien d'ingénieux dans la modélisation RMC - c'est juste une combinaison du premier et du second point.

\section{Remerciements}

The author is extremely grateful to Henry Fischer and Vanessa Coulet for translation of the text into French.

\section{Références}

1. McGreevy R L and Pusztai L 1988 Mol. Sim. 1359.

2. McGreevy R L, Howe M A, Keen D A and Clausen K N 1990 in Neutron scattering data analysis ed. M.W. Johnson, IOP conference series 107165.

3. McGreevy R L and Howe M A 1992 Ann. Rev. Mat. Sci. 22217.

4. McGreevy R L 1995 Nucl. Inst. Meth. in Phys. Res. A 3541.

5. McGreevy R L 1997 in Computer Modelling in Inorganic Crystallography, ed. C.R.A. Catlow Academic Press 151.

6. Gurman S J and McGreevy R L 1990 J. Phys.: Cond. Matter 29463.

7. Keen D A and McGreevy R L 1990 Nature 344423.

8. McKenzie D R, Davis C A, Cockayne D J H, Muller D A and Cassallo A M 1992 Nature $\mathbf{3 5 5} 622$.

9. McGreevy R L, Howe M A and Wicks J D 1993 Studsvik Neutron Research Laboratory internal report. 
10. Keen D A and McGreevy R L 1991 J. Phys.: Cond. Matter 37383.

11. Nield V M, Keen D A and McGreevy R L 1995 Acta Cryst. A 51763.

12. Montfrooij W, McGreevy R L, Hadfield R A and Andersen N-H 1996 J. Appl. Cryst. 29 285.

13. Mellergård A and McGreevy R L 1999 Acta Cryst. A 55783.

14. McGreevy R L 2001 J. Phys.: Cond. Matter 13 R877-913.

15. Metropolis N, Rosenbluth A W, Rosenbluth M N, Teller A H and Teller E 1953 J. Phys. Chem. 211087.

16. Kaplow R, Rowe T A and Averbach B L 1968 Phys. Rev. 1681068.

17. Renninger A L, Rechtin M D and Averbach B L 1974 J. Non-Cryst. Solids 161.

18. Gerold V and Kern J 1987 Acta. Metallurgica 35393.

19. Schweika W and Haubold H-G 1988 Phys. Rev. B 379240.

20. McGreevy R L and Pusztai L 1990 Proc. Roy. Soc. A 430241.

21. Waseda Y, Kang S, Sugiyama K, Kimura M and Saito M 2000 J. Phys.: Cond. Matter 12 195.

22. Bras W, Xu R, Wicks J D, Van der Horst F, Oversluizen M, McGreevy R L and van der Lugt W 1994 Nucl. Inst. Meth. In Phys. Res. A 346394.

23. Wicks J D and McGreevy R L 1997 Phase Transitions 61195.

24. McLaughlin J C 1999 J. Mol. Graphics and Modelling 17275.

25. Wicks J D, Börjesson L, Bushnell-Wye G, Howells W S and McGreevy R L 1995 Phys. Rev. Lett. 74726.

26. Pusztai L and McGreevy R L 2001 Private communication.

27. Kugler S, Pusztai L, Rosta L, Bellissent R and Chieux P 1993 Phys. Rev. B 487685.

28. Keen D A 1997 Phase Transitions 61109.

29. Kohara S 2001 Private communication.

30. Pusztai L and McGreevy R L 1999 J. Neutron Res. 817.

31. McGreevy R L and Pusztai L 1998 Electrochimica Acta 431349.

32. Carlsson P, Swenson J, Börjesson L, Torell L M, McGreevy R L and Howells W S 1998 J. Chem. Phys. 1098719.

33. Karlsson L and McGreevy R L 1995 Solid State Ionics 76301.

34. Evans R A 1990 Mol Sim. 4409.

35. Soper A K 2001 Mol. Phys. in press.

36. Howe M A and McGreevy R L 1991 Phys. Chem. Liq. 241.

37. Colognesi D, de Santis A and Rocca D 1996 Mol. Phys. 88465.

38. Howe M A 1990 Mol. Phys. 69161.

39. Stolz M, Winter R, Howells W S, McGreevy R L and Egelstaff P A 1994 J. Phys.: Cond. Matter 63619.

40. Scheidler M, North A N and Dore J C 1993 Mol. Sim. 11345. 
41. Howe M A, McGreevy R L, Pusztai L and Borzsak I 1993 Phys. Chem. Liq. 25204.

42. Soper A K 1992 in Neutron scattering data analysis ed. M.W. Johnson, IOP conference series 107192.

43. Nield V M, Keen D A, Hayes W and McGreevy R L 1992 J. Phys.: Cond. Matter 46703.

44. Mellergård A 2001 Private communication.

45. Belushkin A V, Kozlenko D P, McGreevy R L, Savenko B N and Zetterström P 1999 Physica B 269297.

46. McGreevy R L and Mellergård A 1999 AIP Conf. series 47919.

47. Mellergård A and McGreevy R L 2000 Chem. Phys. 261267.

48. Andreev Y G and Bruce P G 1998 Mat. Sci. Forum 278-281 14.

49. Tucker M G, Squires M P, Dove M T and Keen D A 2001 J. Phys.: Cond. Matter 13403.

50. Tucker M G, Dove M T and Keen D A 2001 J. Appl. Cryst. submitted.

51. Proffen T and Neder R B 1997 J. Appl. Cryst. 30171.

52. Proffen T 1997 Acta Cryst. 53202.

53. Proffen T 1997 Z. Krist. 212764.

54. Toth G and Baranyai A 1997 J. Chem. Phys. 1077402.

55. Toth G and Baranyai A 1999 Mol. Phys. 97339.

56. Toth $\mathrm{G}$ and Baranyai A 2000 J. Mol. Liq. 853.

57. da Silva F L B 1998 J. Chem Phys. 1092624.

58. Toth G, Pusztai L and Baranyai A 1999 J. Chem. Phys. 1115620.

59. da Silva F L B, Svensson B, Åkesson T and Jonsson B 1999 J. Chem. Phys. 1115622.

60. da Silva F L B, Olivares-Rivas W, Degreve L and Åkesson T 2001 J. Chem. Phys. 114907.

61. Adams S and Swenson J 2000 Phys. Rev. Lett. 844144.

62. Adams S and Swenson J 2001 Phys. Rev. B 63054201.

63. Mellergård A 2001 Private communication.

64. Gehlen P C and Cohen J B 1965 Phys. Rev. A 139844.

65. Kirkpatrick S, Gelatt C D Jr. and Vecci M P 1983 Science 220671.

66. Schommers W 1983 Phys. Rev. A 283599.

67. Levesque D, Weiss J J and Reatto L 1985 Phys. Rev. Lett. 54451.

68. Lyubartsev A B 1995 Phys. Rev. E 523730.

69. Soper A K 1996 Chem. Phys. 202295.

70. Neuefeind J, Fischer H E and Schröder W 2000 J. Phys.: Cond. Matter 128765.

71. Tóth G 2000 J. Mol. Liq. 853.

72. Jenkins T, Greaves G N, Soper A K, Landron C, Hennet L and Coutures J-P $2001 \mathrm{~J}$. Non-Cryst. Solids in press.

73. Car R and Parrinello M 1985 Phys. Rev. Lett. 552471.

74. Foley M, Smargiassi E and Madden P A 1994 J. Phys.: Cond. Matter 65231.

75. Delaye J-M, Ghaleb D, Cormier L and Calas G 2001 J. Non-Cryst. Solids in press. 